\title{
Understanding Visually Induced Motion Sickness through a Homoeopathic Standpoint
}

\author{
Shreyank Kotian \\ Consultant Homeopathic Physician, Dr Kotian's Homoeopathy and Wellness Centre, Mangalore, Karnataka, \\ India.
}

DOI: https://doi.org/10.52403/ijshr.20220125

\begin{abstract}
Motion sickness is one of the most commonly seen discomforts among people that has spread across all age groups and has been affecting people since centuries. It is a phenomenon of sensory conflict between the visual, vestibular and motor system. Visually Induced motion sickness is a sub-type of motion sickness, where the brain interprets sensory messages regarding movement of eyes with respect to the body as inharmonious. Any deviation from the healthy state seen after the exposure to the virtual environment is considered as the symptom of Visually Induced Motion sickness. Nausea is the most disturbing symptom seen in this condition. Along with cold sweating, drowsiness, retching, or vomiting, dizziness or vertigo etc. This phenomenon can be homoeopathically explained as per the classification of diseases by Samuel Hahnemann in different levels. This review summarizes theories behind the causation of visually induced motion sickness, the Homoeopathic basis of its cause and the treatment protocol behind it including a few remedies and Rubrics that may help in clinical practice for the selection of the similimum.
\end{abstract}

Keywords: Visually Induced motion sickness, Homoeopathy, Virtual reality, Cyber-sickness, Simulator sickness

\section{INTRODUCTION}

Visually induced motion sickness (VIMS) is a syndrome that occasionally occurs when physically stationary individuals view compelling visual representations of self-motion. [1]. It is known to appear under visual stimulation such as occurs in driving simulators, flight simulators, movie theatres, or video games [2]. VIMS has been referred to as cyber sickness [3], virtual reality sickness [4], or Cinerama sickness, or simulator sickness [5] based on its different setups where it may occur. VIMS become an important topic to study as it may disrupt the day to day activities of individuals by causing various symptoms that is triggered by the least intense stimuli such as watching an action movie or playing a video game

Benzodiazepines, Anti-cholinergic, Anti-Histamines [7] and specific Homoeopathic remedies like Cocculus indicus [7] have proved to be an effective and established emergency treatment by the different schools of science and medicine. Certain behavioural and environmental modifications has also found to be effective in decreasing the intensity of motion sickness that is determined by the position of head and eyes[8]. But there has been a failure in understanding and removing the trait completely from the individual permanently.

This discussion becomes important due to the changing trends in the entertainment and gaming industries where the world is going into a dependence of virtual reality, simulators and 3D environments. The introduction of an alternate virtual world called the 'METAVERSE' by Mark Zuckerberg's company- Meta is a proof that the future holds enormous scope for the majority of the practices to be run in virtual reality. 
This article is dedicated in understanding the pathophysiological basis of Visually Induced Motion sickness, its homoeopathic and miasmatic basis and treatment protocol as explained in the Organon of Medicine.

\section{EPIDEMOLOGY}

The incidence of VIMS can vary widely, Studies estimate that $60-70 \%$ of all simulator users suffer at least slightly from VIMS and that $80-95 \%$ of users exposed to Virtual Environment show some Motion Sickness-like symptoms[9]. Sherman CR et.al describes how the dropout quota among these users can reach 30\%. [10]

Experimental studies showed that Females have higher incidence of VIMS when compared to men[11,12]. This was further supported by a study that showed a link between intensity of motion sickness and perceived menstrual pain was seen. Differences between women with severe menstrual pain with women with low pain and also when compared to men were found to be significant. [13]

\section{THEORIES REGARDING THE CAUSE OF VIMS [14]} 3.1-SENSORY CONFLICT THEORYThe mismatch between the visual, vestibular, and somatosensory senses is one of the most commonly accepted theories regarding visually induced motion sickness. It occurs when the virtual environment may prompt a bodily movement corresponding to the visual input, but its absence in reality creates a conflict between the expected and experienced stimuli. The vestibular apparatus experiences this, as it is sensitive to the change in velocity and not in a constant position.

\section{2-POSTURAL INSTABLITY THEORY -}

Individuals with lesser postural stability even in constant motion have a higher consequence of VIMS. This occurs in people who have poor postural control even without a visual stimuli in situations like travelling in a vehicle. Hence an experience of a constantly moving visual stimuli will worsen the postural instability that already exists causing VIMS

3.3-EYE MOVEMENTS-According to this, Optokinetic nystagmus (jerk nystagmus induced by full-field visual motion)[15] caused due to the constantly moving visuals can stimulate the vagal nerve causing motion sickness like symptoms. Fixing the vision to one point reducing movements of the eyes has been proved to decrease intensity of VIMS, thus proving the eye movement theory of VIMS

\section{SYMPTOMS OF VISUALLY INDUCED MOTION SICKNESS[14]}

The symptoms related to VIMS do not different much when compared to that of traditional Motion sickness symptoms

Typical symptoms include

- Drowsiness

- Dizziness

- Fatigue

- Pallor

- Cold Sweat

- Occulomotor Disturbances (More prominent than traditional MS)

- Disorientation (More prominent than traditional MS

- Nausea

- Vomiting

It is also noteworthy to understand that neutralising the sensory conflict by a stronger stimuli such as listening to pleasant music [16] or chewing flavoured gum[17] and also having a stronger olfactory stimuli [18] has found to reduce the intensity of motion sickness and its symptoms.

\section{SCALES TO MEASURE VISUALLY INDUCED MOTION SICKNESS}

Several scales have been developed and used efficiently in identifying the presence of and measuring the intensity of visually induced motion sickness. These scales have been developed after series of experiments that has confirmed its validity across the years. Revised versions of the already existing scales have also been 
developed to keep in pace with the changing trends.

Few of the scales that may be used to measure the intensity of VIMS are-

\section{1-Simulator Sickness Questionnaire} (SSQ)-[19] This scale derived from the Pensacola Motion Sickness Questionnaire (MSQ), This is a 16-item scale that measures the symptoms in 3 groups-Nausea, Occulomotor disturbances and disorientation which are not independent to each other. Each of these groups describe symptoms correspondingly that can measure the intensity of each sign independently and in dependence to the other $[\mathbf{2 0 , 2 1 ]}$

\section{2-Visually Induced Motion Sickness Susceptibility Questionnaire (VIMSSQ) [12] ${ }^{-}$It is a modification of the Motion Sickness Susceptibility Questionnaire which was unique than other scales as it was able to predict individual susceptibility to motion sickness and not only measure the intensity of symptoms. Scores on the VIMSSQ are measured on basis of presence of symptoms of nausea, headache, fatigue, dizziness, and eyestrain in a virtual environment based on prior experiences.}

5.3-Other scales -Fast Motion sickness Scale (FMS)[22], Virtual reality sickness questionnaire (VRSQ) [23], Revised Simulator Sickness Questionnaire (SSQ) [24]

\section{HOMOEOPATHIC PERSPECTIVE 6.1-Organon of medicine and VIMS[25]}

When we try to describe the Homoeopathic basis of any diagnosis, it is apt to classify it accurately under the Hahnemann's classification of Diseases as explained in the Organon of medicine.

\subsection{1-VIMS as an indisposition}

Aphorism 150 states - "If a patient complains of one or more trivial symptoms that have been only observed a short time previously, the physician should not regard this as a fully developed disease that requires serious medical aid. A slight alteration in the diet and regimen will usually suffice to dispel such an indisposition"

This may only explain VIMS symptoms on persons who has had no previous exposure to the virtual environment and produce such symptoms only in that condition. If the VIMS symptoms are removed after the removal of the Visual stimuli, it may be considered as the Indisposition of the Individual. Although, it is acceptable only in cases where this stimuli is a onetime occurrence for the individual and will not be suitable in cases where the visual stimuli is a daily activity for any individual like professional gamers or Animators. Hence, the treatment protocol of indisposition where no medicines are given and the stimuli is removed may help in decreasing the symptoms at that moment, but would not be suitable in removing the trait from the individual.

\subsection{2-VIMS as an individual acute disease / one sided disease}

In Aphorism 5, Hahnemann says "Useful to the physician in assisting him to cure are the particulars of the most probable exciting cause of the acute disease...."Considering the theories of VIMS, Several exciting causes may exist that can cause sensory conflict or troubles with eye movements. Hence remedies that can match with the symptoms as well as the cause, maybe given as an acute remedy.

Here the treatment based on the cause becomes the primary focus which may remove the symptoms respectively. The acute manifestations most of the times, if not always, are the transient explosion of the latent miasm. Hence, although the treatment with homeopathic remedies based on acute totality maybe enough to remove symptoms, recurrence of the symptoms or even the removal of that trait completely with an anti miasmatic remedy.[26]

\subsection{3-VIMS as a True Chronic disease}

Hahnemann in his work- Chronic diseases, explained about the 3 major 
classification of chronic diseases that he could arrive at after more than a decade of observation and experimentation. [27] The three chronic miasms that Hahnemann introduced in 1828 were called Syphilis (the chancre miasm), Sycosis (the gonorrhoeal miasm) and Psora (the itch miasm). He mentioned clearly that out of the three, only Psora was the non-venereal miasm and the other two developed from the ill effects of Syphilis and Gonorrhoea respectively.

We know that The Miasmatic diagnosis of any condition will depend on the stage of the disease and its underlying cause. All the causes of VIMS point towards a complete physiological entity with no structural causes involved, which points its direction towards Psora. Although, this will also depend on the stage of this disease

H.A.Roberts in his book -The principles and Art of Cure by Homoeopathy under the chapter-DISEASE CLASSIFICATION: PSORA contd. Speaks about how Psora is never the basis of a structural change alone [28]. It may be seen only when conjoined with other miasms. This gives a stronger basis for Motion sickness, which is pure physiological phenomenon with no structural change involved, A Psoric trait. He also explains how Psora has- "PSORA has numerous sensations of vertigo...and are often induced or aggravated by emotional disturbances. Hahnemann speaks of the vertigo of Psora as being many and peculiar, brought on by walking, motion, looking up and quickly, rising form sitting or lying; bilious vertigo, floating, from digestive disturbances, with spots before the eyes; desire to keep quiet by lying down, which >. In this desire to lie down and > by lying down we have the outstanding characteristic of the whole underlying condition" Here it clearly shows how vertigo is induced by various motion, including that of only the eye and ameliorated by solving the sensorial conflict.

We know that the symptoms of
VIMS include Dizziness involving

disorientation, headache and blurriness. These symptoms are included under the Psoric miasm by ML Tylor as -vertigo with eructation, with rush of blood to the head, or face, with headaches, prosopalgia followed with temporary blindness [29]

Hence, an anti-Psoric remedy given infrequently after the complete commencement of the acute attack may help in reducing the reoccurrence and also in removing the trait from the individual as a whole.

\section{2-REMEDIES RELATED TO VISUALLY INDUCED MOTION SICKNESS}

This is section, remedies only related to symptoms of VIMS are mentioned and not those of Motion sickness in general. This is specifically targeted to help the physician treat symptoms based on it aggravated or caused by the movement/ motion of eyes in general. This section is divided based symptoms of vertigo, nausea, headache and disorientation and the rubrics /remedies in the corresponding repertories

\subsection{1-REPERTORY OF HERING'S GUIDING SYMPTOMS OF OUR MATERIA MEDICA BY CALVIN B. KNERR, M. D.[30,31] VERTIGO}

- Looking around, when: - Con.

- Looking down or turning, when: - Spig.

- Looking long and steadily, on: - All-s.

- Looking at objects in rapid motion, when: - Sulph.

HEADACHE-

- Eyes, better while, are steadily fixed upon one object and while patient is thinking of one subject: - Sabad.

- Moving, worse, eyes: -Bell., Cimic., Coloc., Hep., Mur-ac., Ptel., Sep., Sil.

- Moving, worse, or turning up eyes: Bapt.

- Turning, worse, eyes: - Sep.

- NAUSEA, looking at moving objects, in (hyperopia cum asthenopia):- Jab. 
- SEASICKESS-Motion, worse from least bodily, better on deck and fresh cold air: - Tab.

\subsection{2- REPERTORY OF THE HOMOEOPATHIC MATERIA MEDICA- J.T.KENT[32] VERTIGO}

- LOOKING with eyes turned: Spig.

- Looking at moving object, at: Agar., anac., con., cur., graph., jab., 1 aur., mosch., nat-m., olnd., sep., sulph.

- Looking with eyes turned steadily- amel. : Dig., sabad.

- MOTION, eyes: Bell., chel., cocc., con., mur-ac., petr., plat., puls., spig

- Motion, from, eyes, of- Asar.

STOMACH, Nausea, using the eyes- Ther, Con, Graph, Jeb, Sep

6.2.3 BOGERBOENNINGHAUSEN'S CHARACTERI STICS AND REPERTORY[33]

- Vertigo, aggravation, moving- Chel. Con

- Vertigo, aggravation, eyes, turningSpig, Plat

\section{SUGGESTIONS}

Research studies conducted in conjunction with the fraternity dealing with virtual environments and Simulators would help understand the action of various remedies more clearly with respect to the changing trends. A long-term study to prove if the trait of motion sickness can completely remove in a an individual permanently through homoeopathy would not only be beneficial to the individual to adapt to the future of simulators, but also will help to prove the basis of Homoeopathic science of permanent and gentle cure.

\section{CONCULSION}

Over the years, not only has the homoeopathic system of medicine proved its permanent action of conditions that has temporary scope in few conditions, but also has shown its vastness of scope in being ready to treat conditions that newly emerge in the ever changing future. It is up to the Homoeopathic physician to understand how to use the tool to treat and prevent diseases that can affect the daily life of individuals, improve their quality of life and be beneficial to the society as a whole.

Acknowledgement: None

\section{Conflict of Interest: None}

\section{Source of Funding: None}

Ethical Approval: Not Applicable

\section{REFERENCES}

1. Keshavarz B, Hecht H, Lawson BD. Visually induced motion sickness: causes, characteristics, and countermeasures. Handbook of virtual environments CRC Press. 2014 Sep 10 (pp. 652-703).

2. Hettinger LJ, Riccio GE. Visually induced motion sickness in virtual environments. Presence: Teleoperators \& Virtual Environments. 1992;1(3):306-10.

3. Kennedy RS, Lanham DS, Drexler JM, Massey CJ, Lilienthal MG. A comparison of cybersickness incidences, symptom profiles, measurement techniques, and suggestions for further research. Presence: Teleoperators \& Virtual Environments. 1997 Dec;6(6):638-44..

4. Johnson DM. Introduction to and review of simulator sickness research. Army research inst field unit fort rucker al; 2005 apr 1.

5. Miller II EF, Graybiel A. Motion sickness produced by head movement as a function of rotational velocity. Naval Aerospace Medical Institute, Naval Aerospace Medical Center; 1970.

6. Kumar GP, Anilakumar KR, Mallesha YC, Sharma RK. motion sickness: manifestations and Prevention. Defence Life Sci. J.. 2020;5(3):230-7.

7. REBER A, LEROY MH, POITEVIN B. Visuo-vestibular reflexes adjustment by GABA antagonists. InHigh Dilution Effects on Cells and Integrated Systems 1998 (pp. 259-262).

8. Leung AK, Hon KL. Motion sickness: an overview. Drugs in context. 2019;8.

9. Stanney KM, Mourant RR, Kennedy RS. Human factors issues in virtual environments: A review of the literature. Presence. 1998 Aug;7(4):327-51. 
10. Sherman CR. Motion sickness: review of causes and preventive strategies. Journal of travel medicine. 2002 Sep 1;9(5):251-6.

11. Flanagan MB, May JG, Dobie TG. Sex differences in tolerance to visually-induced motion sickness. Aviation, space, and environmental medicine. 2005 Jul 1;76(7):642-6.

12. Keshavarz B, Saryazdi R, Campos JL, Golding JF. Introducing the VIMSSQ: Measuring susceptibility to visually induced motion sickness. InProceedings of the Human Factors and Ergonomics Society Annual Meeting 2019 Nov (Vol. 63, No. 1, pp. 22672271). Sage CA: Los Angeles, CA: SAGE Publications.

13. Hemmerich WA, Shahal A, Hecht H. Predictors of visually induced motion sickness in women. Displays. 2019 Jul 1;58:27-32.

14. Keshavarz B, Riecke BE, Hettinger LJ, Campos JL. Vection and visually induced motion sickness: how are they related?. Frontiers in psychology. 2015 Apr 20;6:472.

15. 15.Furman J. Optokinetic Nystagmus. In: J. Aminoff M, B. Daroff R, ed. by. Encyclopedia of the Neurological Sciences. 2nd ed. Academic Press, Elsevier; 2014.

16. Keshavarz B, Hecht H. Pleasant music as a countermeasure against visually induced motion sickness. Applied ergonomics. 2014 May 1;45(3):521-7

17. Kaufeld M, De Coninck K, Schmidt J, Hecht H. Chewing gum reduces visually induced motion sickness. Experimental brain research. 2022 Jan 7:1-3.

18. Ranasinghe N, Jain P, Tolley D, KarwitaTailan S, Yen CC, Do EY. Exploring the Use of Olfactory Stimuli Towards Reducing Visually Induced Motion Sickness in Virtual Reality. InSymposium on Spatial User Interaction 2020 Oct 31 (pp. 1-9)

19. Kennedy RS, Lane NE, Berbaum KS, Lilienthal MG. Simulator sickness questionnaire: An enhanced method for quantifying simulator sickness. The international journal of aviation psychology. 1993 Jul 1;3(3):203-20.

20. Dużmańska N, Strojny P, Strojny A. Can simulator sickness be avoided? A review on temporal aspects of simulator sickness. Frontiers in psychology. 2018 Nov 6;9:2132

21. Balk SA, Bertola DB, Inman VW. Simulator sickness questionnaire: twenty years later.
22. Keshavarz B, Hecht H. Validating an efficient method to quantify motion sickness. Human factors. 2011 Aug;53(4):415-26.

23. Kim HK, Park J, Choi Y, Choe M. Virtual reality sickness questionnaire (VRSQ): Motion sickness measurement index in a virtual reality environment. Applied ergonomics. 2018 May 1;69:66-73.

24. Sevinc V, Berkman MI. Psychometric evaluation of Simulator Sickness Questionnaire and its variants as a measure of cybersickness in consumer virtual environments. Applied ergonomics. 2020 Jan 1;82:102958.

25. Hahneman S. Organon of Medicine (5th and 6th Edition). Translated by Dudgeon RE \& Boeriche W. New Delhi: B.

26. Sarkar BK. Hahnemann's Organon of Medicine. Calcutta: M. Bhattacharya \&co.(P) ltd. Publications. 2005.

27. Hahnemann S. The Chronic Disease, Their Peculiar Nature and Their Homœopathic Cure. Translated by Tafel LH [1895]. Indian Reprint, New Delhi: B.

28. 28. Robert HA. The principles and art of cure by Homoeopathy. 3rd reprint ed. New: B Jain Publishers Pvt. Ltd. 2007.

29. Tyler M. A Comparison of Chronic Miasms by Tyler, ML [Internet]. HomeopathyBooks.in. [cited 2 February 2022]. Available from: https://homeopathybooks.in/a-comparison-ofchronic-miasms-by-tyler-ml/

30. Knerr CB, Hering C. A Repertory of Hering's Guiding Symptoms of our Materia Medica. FA Davis; 1896.

31. Hering C. Herings Guiding Symptoms of Our Materia Medica. B. Jain Publishers; 2003 Jun 30 .

32. Kent JT. Repertory of the homoeopathic materia medica. B. Jain Publishers; 1992.

33. Boger CM. Boenninghausen's characteristics materia medica \& repertory with word index. B. Jain Publishers; 2002.

How to cite this article: Shreyank Kotian. Understanding visually induced motion sickness through a homoeopathic standpoint. International Journal of Science \& Healthcare Research. 2022; 7(1): 148-153. DOI: https:// doi.org/10.52403/ijshr.20220125 